YITP-SB-02-80

January 23, 2003

\title{
Approaching the Final State in Perturbative QCD
}

\author{
George Sterman \\ C.N. Yang Institute for Theoretical Physics \\ Stony Brook University, State University of New York \\ Stony Brook, New York 11794 - 3840, U.S.A.
}

\begin{abstract}
Infrared safe differential cross sections, such as event shape distributions, can be measured over wide kinematic ranges, from regions where fixed order calculations are adequate to regions where nonperturbative dynamics dominate. Such observables provide an ideal laboratory for the study of the transition between weak and strong coupling in quantum field theory. This talk begins with some of the fundamentals of the perturbative description of QCD and the basis of resummation techniques, followed by a brief discussion of selected topics from recent fixed-order and resummed calculations. It focuses on how resummed perturbation theory has been used to deduce the structure of nonperturbative corrections, and to provide a framework with which to address the transition from short- to long-distance dynamics in QCD.
\end{abstract}

\section{Introduction}

Quantum chromodynamics (QCD) serves both as tool and background for new physics searches [1]. Control over QCD corrections is crucial for the interpretation of any experiment in which hadronic matter appears in the initial or final states, or even (as in the measurement of the muon's magnetic moment) only in virtual states. In addition, QCD is fascinating in its own right as a quantum field theory. It combines strong and weak coupling dynamics in any high energy experiment that is sensitive to momentum transfers much beyond the scale of the strong coupling, $\Lambda_{\mathrm{QCD}}$, and every experiment that probes higher energies encodes the transition between partonic degrees of freedom at short distances to hadronic degrees of freedom at long.

This talk approaches these issues from the partonic, short distance side, through the study of perturbation theory. I will argue that perturbative QCD has much to say about the structure of the larger theory that contains it. The discussion begins with a sketch of basic concepts of perturbative QCD, followed by a review of some 
important recent developments at finite order. The extension of perturbative methods to multiscale observables requires resummation, and I will describe how resummed perturbation theory encodes nonperturbative information, with the operator product expansion as the classic example. Analogous reasoning is extended to jet-related cross sections, and examples are given of the interesting results that emerge from such an analysis.

\section{Some Concepts in Perturbative QCD}

\subsection{Asymptotic freedom and infrared safety}

The characteristic feature of $\mathrm{QCD}$ is its asymptotic freedom, in which the coupling becomes weaker when it is measured at shorter distances. This remains a purely abstract attribute, however, until we identify observable quantities that can be expanded in the coupling with finite coefficients. Such quantities are said to be infrared safe [2]. A few cross sections, such as the total and suitably-defined jet cross sections in $\mathrm{e}^{+} \mathrm{e}^{-}$ annihilation, are infrared safe, and may be expanded as

$$
\begin{aligned}
Q^{2} \sigma_{\mathrm{phys}}(Q) & =\sum_{n} c_{n}\left(Q^{2} / \mu^{2}\right) \alpha_{s}^{n}(\mu)+\mathcal{O}\left(\frac{1}{Q^{p}}\right) \\
& =\sum_{n} c_{n}(1) \alpha_{s}^{n}(Q)+\mathcal{O}\left(\frac{1}{Q^{p}}\right)
\end{aligned}
$$

where $p$ is some integer and $\mu$ is a renormalization scale. In the second expression we have used the renormalization group invariance of physical cross sections.

\subsection{Factorization}

Cross sections for processes with hadrons in the initial state are not by themselves infrared safe. Nevertheless, if they involve heavy particle production, or more generally involve a large momentum transfer, $Q$, then short- and long-distance components of the cross section can be factorized [3], usually into a convolution form,

$$
Q^{2} \sigma_{\mathrm{phys}}(Q, m)=\omega_{\mathrm{SD}}\left(Q / \mu, \alpha_{s}(\mu)\right) \otimes f_{\mathrm{LD}}(\mu, m)+\mathcal{O}\left(\frac{1}{Q^{p}}\right)
$$

In this expression, $\otimes$ represents a convolution, most familiarly in terms of partonic momentum fractions, and $\mu$ is a factorization scale, separating the short-distance dynamics of the hard subprocess, in $\omega_{\mathrm{SD}}$, from the long-distance dynamics in $f_{\mathrm{LD}} \cdot f_{\mathrm{LD}}$ depends on various infrared, often nonperturbative scales, denoted $m$. For inclusive leptonhadron deep-inelastic scattering (DIS), in which $Q$ is the momentum transfer, and for various other inclusive and semi-inclusive hadronic hard-scattering cross sections, $f_{\mathrm{LD}}$ represents products of parton distribution functions. For single-particle inclusive cross 
sections, it includes fragmentation functions. Generally speaking, 'new physics' is part of the short distance functions $\omega_{\mathrm{SD}}$, while the long-distance $f_{\mathrm{LD}}$ 's are universal among processes, making predictions possible on the basis of hypothetical couplings to new particles or rare processes at short distances. Factorizations also apply to many scattering amplitudes and forms factors, of which those involving the decay of B mesons have attracted interest recently [4].

Eq. (2) applies not only to factorized cross sections with hadrons in the initial state, but also to many infrared-safe jet cross sections, for which the scale $m$ is large enough to be perturbative, but is still much smaller than the highest scale $Q: Q \gg m \gg \Lambda_{\mathrm{QCD}}$. An example is the limit of 'narrow jets' in $\mathrm{e}^{+} \mathrm{e}^{-}$annihilation, where $m$ may be taken as the mass of the jet [5].

\subsection{Factorization proofs and resummation}

The proofs of relations like Eq. (2) are all based on the observation that short-distance processes, organized within the function $\omega_{\mathrm{SD}}$, are quantum-mechanically incoherent with dynamics at much longer length scales. This is the same observation that makes possible the operator product expansion. Extensions of Eq. (2) to jet cross sections and various decay and elastic amplitudes depend on the further observation that there is a mutual incoherence in the dynamics of particles receding from each other at the speed of light. Often, these factorization relations hold to all orders in perturbation theory and to all powers of $\ln (\mu / Q)$ [6]. Interest in these issues has reemerged in analyses of high energy processes in the language of effective theories [7].

Whenever there is factorization, there is some form of evolution and resummation. To see why, we observe that a physical cross section cannot depend on the factorization scale,

$$
0=\mu \frac{d}{d \mu} \ln \sigma_{\text {phys }}(Q, m) .
$$

From this it follows that the variations of long-distance and short-distance functions with respect to $\mu$ must compensate each other, through a function of the variables that they hold in common:

$$
\mu \frac{d}{d \mu} \ln f_{\mathrm{LD}}(\mu, m)=-P\left(\alpha_{s}(\mu)\right)=-\mu \frac{d}{d \mu} \ln \omega_{\mathrm{SD}}\left(Q / \mu, \alpha_{s}(\mu)\right) .
$$

In deep-inelastic scattering, and other inclusive hard-scattering cross sections, where $\omega_{\mathrm{SD}}$ and $f_{\mathrm{LD}}$ are connected by a convolution in momentum fractions, the functions $P\left(z, \alpha_{s}\right)$ are the DGLAP splitting functions [8].

By the same token, wherever there is evolution, there is a resummation of perturbation theory, generated by solving the evolution equation. Again thinking of DIS, we may fix $\mu=Q$ in Eq. (2), compute $\omega_{\mathrm{SD}}$ as a power series in $\alpha_{s}(Q)$, and derive the 
$Q$-dependence of the cross section by solving (4) for the functions $f_{\mathrm{LD}}(Q, m)$ :

$$
\begin{aligned}
\ln \sigma_{\mathrm{phys}}(Q, m) \sim \omega_{\mathrm{SD}}\left(1, \alpha_{s}(Q)\right) & \\
& \otimes \exp \left\{\int_{q}^{Q} \frac{d \mu^{\prime}}{\mu^{\prime}} P\left(\alpha_{s}\left(\mu^{\prime}\right)\right)\right\} f_{\mathrm{LD}}(q, m) .
\end{aligned}
$$

The most impressive successes of this approach are in observables with a single hard scale. Inclusive DIS is the exemplary case, as illustrated in Fig. 1 for the structure function $F_{2}(x, Q)$, which is proportional to the cross section to produce an hadronic final state of total invariant mass squared equal to $Q^{2}(1-x) / x$, at momentum transfer $Q$.

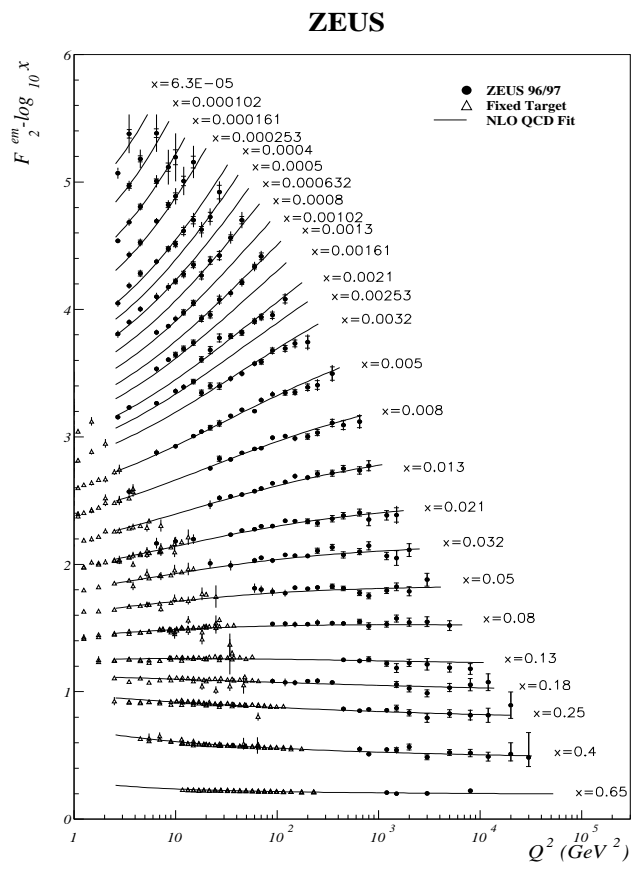

Figure 1: Data for $F_{2}(x, Q)$ with QCD fit based on evolution, from Ref. [9].

As mentioned above, however, only a limited number of observables depend on a single hard scale. A more typical example is the distribution of $\rho=\left(m_{J} / Q\right)^{2}$, from $\mathrm{e}^{+} \mathrm{e}^{-}$annihilation dijet events, with $m_{J}$ the mass of the heavier jet in the center-of-mass (c.m.). The experimental distribution in $\rho$ at the $\mathrm{Z}$ pole is illustrated in Fig. 2, taken from [10]. In this case, the two scales are the c.m. energy, $Q=\sqrt{s}$ and the mass of the 'heavy' jet. The data is shown as a function of $\rho=m_{J}^{2} / Q^{2}$, and even resummed perturbation theory (next-to-leading logarithm in $m_{J} / Q$ ) is not adequate to follow it. It is necessary to include power corrections in the lighter scale: $1 / m_{J}^{p}$ (as organized in a shape function, see below), which become more and more important in the 'exclusive 
limit' of light jet masses. Notice that most events are in this range; going one step beyond the fully inclusive cross section, we are required to find a description with nonperturbative as well as perturbative input, even though the observable in question is infrared safe. As we decrease $m_{J}$, nonperturbative effects vary continuously from a few to tens of percent. Thus, within this single set of data, at a single energy, we can select events whose formation is sensitive to an adjustable mix of perturbative and nonperturbative dynamics. This is an ideal testing-ground for quantum field theory.

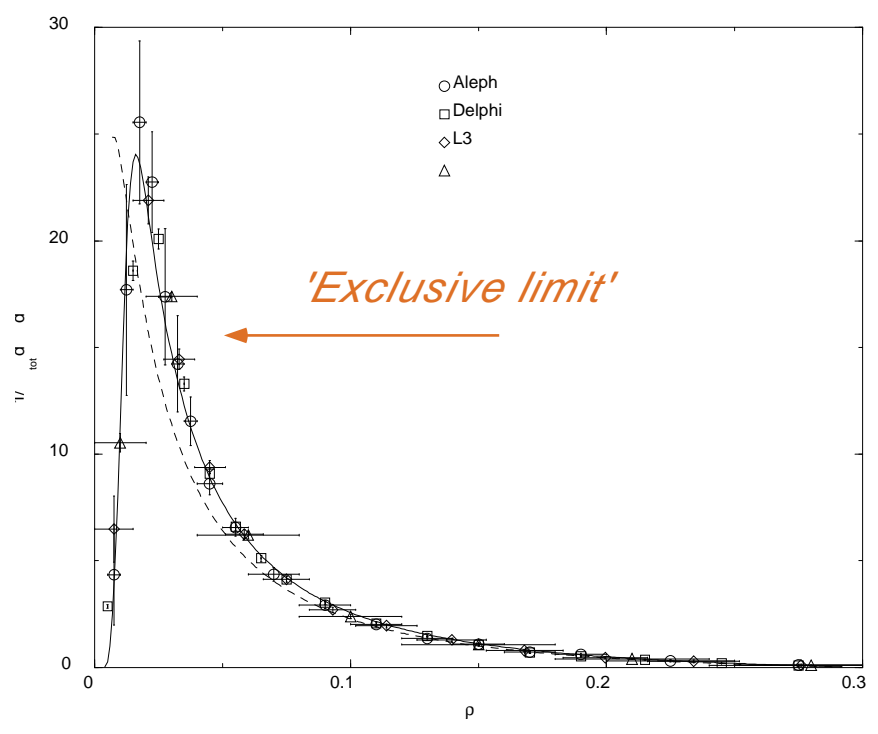

Figure 2: Heavy jet distribution at the $\mathrm{Z}$ pole, from Ref. [10]. The dashed line is resummed perturbation theory, and the solid line a fit with a shape function.

I shall discuss below recent attempts to organize nonperturbative corrections to infrared safe cross sections starting with perturbation theory, in a manner similar to factorization theorems like Eq. (2). To do so with confidence, we should attempt to organize perturbation theory to all orders in the coupling, the level at which we understand Eq. (2). First, however, let us touch on some of the very substantial progress of the past few years that is based on finite-order calculations.

\section{Toward a Two-Loop Phenomenology}

Fig. 3 is a cartoon of the progress of fixed-order calculations in perturbative QCD over the past three decades, with tree amplitudes under control around 1980, one-loop amplitudes around 1990, and two-amplitudes since 2000 (early two-loop advances were reported [11], with recent reviews in [12]). In the years following their calculation, amplitudes at the tree and one-loop level were implemented into factorized cross sections 
like (2). With the hard-scattering computed at tree level, the cross section is termed 'leading order' (LO). At one loop, it is 'next-to-leading order' (NLO), and so on. Now we are at the dawn of an age of NNLO. It is still the dawn, because loop diagrams suffer from various infrared divergences, which must be controlled by adding gluon emission diagrams and constructing useable, factorized hard-scattering functions. It is a big step from a set of (dimensionally) regulated amplitudes to a factorized cross section (2) that can be compared to experiment. Still, with the two-loop amplitudes in hand, the goal of NNLO phenomenology is certainly attainable.

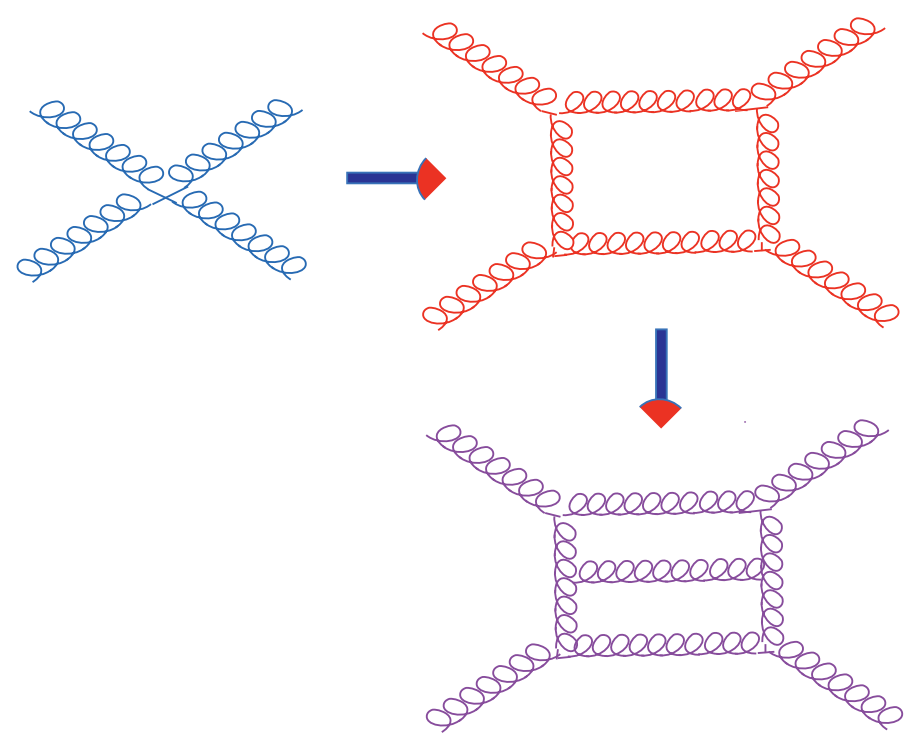

Figure 3: Progress in low-order calculation.

Why go to the trouble of these very challenging calculations? With $\alpha_{s}(Q) \sim 0.1$ at $Q \sim 100 \mathrm{GeV}$, computations to order $\alpha_{s}{ }^{2}$ can reach the accuracy of one percent, an admirable goal in its own right for the strong interactions. At the same time, accuracies of a few percent will enable a new class of investigations of QCD, by covering kinematic regions where nonperturbative power corrections vary from a few percent to tens of percent, as we have seen above. Beyond this, one percent accuracy will make possible a new generation of determinations of the strong coupling itself, the uncertainty in whose precise value is a major limitation on our ability to extrapolate the standard model into the realm of new physics [13]. Recent mileposts on this road include the calculation of $\mathcal{O}\left(\alpha_{s}{ }^{3}\right) 3$-jet amplitudes in electron-positron annihilation [14].

For factorizable jet and other cross sections at $n$ loops in the hard scattering, it is desirable to have $n+1$ loops in the splitting function $P\left(z, \alpha_{s}\right)$, in Eq. (4). Progress toward $P\left(z, \alpha_{s}\right)$ to $\alpha_{s}^{3}$ has included numerical estimates [15], leading to studies of deepinelastic scattering at this level [16], as well as a growing development of the methods 
necessary for full analytic expressions [17] at three loops. The coming decade should see the full realization of the second step of Fig. 3.

The long-distance components of factorized cross sections are parton distributions (PDFs) and fragmentation functions. Unpolarized parton distributions implemented with NLO calculations of hard scattering have reached a stage of refinement over large ranges of parton fraction $x$. These refinements are of great significance, because they are a limiting factor in our ability to extrapolate known cross sections to the scales at which new physics signals may appear. This said, there have still been 'surprises', in just the past few years, such as the unexpected behavior of sea antiquarks as measured by experiment E866 at Fermilab [18], and promptly incorporated into the following round of parton distribution functions $[19,20]$.

Perhaps the most important recent development in the technology of PDFs is the effort to quantify their uncertainties [21]. This is a daunting task, because any set of PDFs for all of the quarks, antiquarks and the gluon requires the specification of a substantial set of parameters. Generally, in a 'global' fit, these parameters are fixed by comparison with a set of existing data. Any estimate of the uncertainty in a calculated process depends in a unique way on all of these parameters, each of which is sensitive to both statistical and systematic errors in the original data set. What we are interested in is not so much the uncertainties in the PDFs themselves, but what these uncertainties mean for cross sections that we would like to calculate. To determine this, it is necessary to specify, not just a 'best fit' for the PDFs, but a set of degrees of freedom accompanied by correlated error estimates. With this information, it is possible to create an ensemble of PDFs, selected according to their statistical likelihood. Each element of such an ensemble provides an independent calculation of a factorized cross section (2), and the distribution of these results determines the desired uncertainty. Needless to say, this procedure is computationally-intensive in all of its variants, depending on the choices of parameterization, the analysis of parameter uncertainty, and the implementation of the ensemble-creation process. The rewards, however, are substantial, and the effort is mandatory for extrapolation to the range of the highest-energy hadron accelerators.

\section{Resummation}

Fixed-order calculations work best for single-scale observables, with the jet cross sections in hadronic collisions offering a fine example, both of the range of applicability, and the uncertainties involved [22]. The cross section for the production of bottom quarks is an example involving more controversy, and possible recent progress [23]. Any finite-energy calculation, however, leaves the nagging question of what happens at yet higher order. For single-scale inclusive cross sections, the comparison of LO with NLO and NNLO, when available, can give confidence or shake it. (See, for example, recent NNLO calculations of inclusive Higgs production at hadron colliders [24].) Nev- 
ertheless, there are certain general features of perturbative corrections that demand study at all orders, even in one-scale cross sections, and in most cases two-scale cross sections cannot be controlled at all without a classification and summation of such corrections at all orders. Examples of these two possibilities are threshold and $Q_{T}$ resummation.

\subsection{Threshold resummation}

Threshold resummation addresses a specific set of corrections at all orders in perturbation theory for factorized cross sections. Consider a generic factorized cross section for the inclusive production of an observable final-state object $F$ of invariant mass $Q$, written in terms of partonic fractions as

$$
Q^{2} \sigma_{A B \rightarrow F}(Q)=f_{a / A}\left(x_{a}\right) \otimes f_{b / B}\left(x_{b}\right) \otimes \omega_{a b \rightarrow F+X}\left(z=\frac{Q^{2}}{x_{a} x_{b} S}\right),
$$

with the $f_{c / H}$ PDFs for parton $c$ in hadron $H$. The hard-scattering cross section depends on variable $z$, which is the ratio of $Q^{2}$ to the squared invariant mass of the pair of partons that initiate the process $a b \rightarrow F+X$, where $X$ denotes additional radiation in the inclusive final state. Of special interest is the limit $z \rightarrow 1$, which is called partonic threshold. At partonic threshold, the partons $a$ and $b$ have only enough energy to produce $F(Q)$, with nothing left over for radiation. This is a special limit in field theory, because a hard scattering favors processes with copious radiation. The consequence of this mismatch is that $z=1$ is associated with singular but integrable distributions, such as

$$
\omega_{a b \rightarrow F}^{(r)}(z) \sim\left(\frac{\alpha_{s}}{\pi}\right)^{r} \frac{1}{r !}\left[\frac{\ln ^{2 r-1}(1-z)}{1-z}\right]_{+},
$$

where a plus distribution $[g(x)]_{+}$with $g(x)$ singular at $x=1$ is defined (like a delta function) by its integrals with smooth functions:

$$
\int_{y}^{1} d x[g(x)]_{+} f(x)=\int_{y}^{1} d x g(x)[f(x)-f(1)]-f(1) \int_{0}^{y} d x g(x) .
$$

In Eq. (6), the singular distributions are in $\omega_{a b \rightarrow F+X}(z)$, which has the information on perturbative radiation, and the smooth functions are the PDFs. While all of the plus distributions are integrable, they become more and more singular at higher orders, and it is natural to try to estimate their overall effect beyond the lowest orders.

We are able to resum singular plus distributions because at partonic threshold the hard-scattering process returns to the elastic limit that we have referred to above, in which final state radiation is grouped into well-collimated jets, associated either with the fragments of the incoming hadrons, or with a set of nearly light-like outgoing jets. Such a momentum configuration is illustrated by Fig. 4 for the production of two jets. 


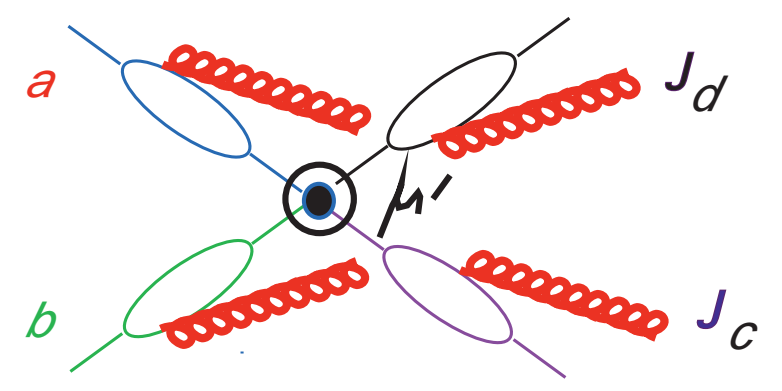

Figure 4: Elastic kinematics and refactorization scale at partonic threshold.

At partonic threshold, we may implement the sequence of reasoning that we described in Sec. 2. Quanta are part of well-collimated jets, moving relatively at the speed of light, or are very soft. We may (re)factorize the partonic cross section in this limit, as indicated in Fig. 4 by the circle and 'refactorization scale', $\mu^{\prime}$, which leads to new evolution equations, and hence to resummation. For the hard scattering of Eq. (6), we find expressions whose expansion generates the terms identified in Eq. (7) to all orders in perturbation theory [25]. The resummation is most conveniently exhibited in the space of Mellin moments of $\omega_{a b \rightarrow F+X}$, where they exponentiate as

$$
\begin{aligned}
& \int_{0}^{1} d z z^{N-1} \omega_{a b \rightarrow F+X}(z) \\
& \sim \exp \left[-\sum_{i=a, b} \int_{0}^{1} d z \frac{z^{N-1}-1}{1-z} \int_{(1-z)^{2} Q^{2}}^{Q^{2}} \frac{d m^{2}}{m^{2}} A_{i}\left(\alpha_{s}(m)\right)\right] \\
& \sim \exp \left[\sum_{i=a, b} \int_{Q^{2} / N^{2}}^{Q^{2}} \frac{d m^{2}}{m^{2}} A_{i}\left(\alpha_{s}(m)\right) \ln \left(\frac{N m}{Q}\right)\right]
\end{aligned}
$$

in terms of anomalous dimensions $A_{i}\left(\alpha_{s}\right)=C_{i}\left(\alpha_{s} / \pi\right)+\ldots$, with $C_{i}=C_{F}$ for $i=q, \bar{q}$ and $C_{A}$ for gluons. Eq. (9) generates the leading logarithms at each order; the complete expression involves process-dependent nonleading logs. At each order in the exponent, one of the logarithms can be evaluated explicitly, as shown, but in the remaining integral the strong coupling runs down to arbitrarily low scales. We will return to this intriguing feature of the resummed cross section below. There are various ways of dealing with this low-scale limit [26, 27, 28, 29], and although the issue is far from settled, the bottom line seems to be that the effect of singular distributions at partonic threshold is a modest enhancement, along with a reduction of dependence on the factorization scale. 


\subsection{Color flow in resummation and at fixed order}

The expression (9) involves radiation that is nearly collinear to the incoming and outgoing partons at the hard scattering, and is completely incoherent among these sets of lines. It organizes the leading logarithms in $N$, and a portion of subleading corrections. Beyond leading logarithm, however, the active partons also radiate soft gluons coherently, in a manner that depends on the exchange of color at short distances schematically, inside the dashed circle labelled $\mu^{\prime}$ in Fig. 4. At this level, threshold resummation has an interesting interplay with the one- and two-loop calculations $[11,30,31]$ represented by Fig. 3 .

In the resummed cross section, the effects associated with coherent soft-gluon emission are single-logarithmic in the moment space of (9),

$$
\exp \left[\int_{Q / N}^{Q} \frac{d m}{m} \lambda^{(\mathrm{f})}\left(\alpha_{s}(m)\right)\right],
$$

where the $\lambda$ 's are eigenvalues of a set of anomalous dimension matrices, which can be determined by separation of variables as in Eq. (4). They are matrices because the short-distance function of Fig. 4 (inside the circle) can be thought of as a vector in the space of color exchange. For example, when the active partons are all gluons, there are as many as eight independent color exchanges at short distances. As $\mu^{\prime}$ changes, gluons that are exchanged near scale $\mu^{\prime}$ enter or leave the hard scattering. These effects are reflected in the NLO amplitudes of Fig. 3, and influence the infrared structure of NNLO in an essential way $[30,31]$.

A striking example is the $g g \rightarrow g g$ anomalous dimension matrix, which controls single logarithms in threshold resummation for gluonic jet production [32], with $N_{c}$ the number of colors:

$$
\left(\begin{array}{ccccccccc}
T & 0 & 0 & 0 & 0 & 0 & -\frac{U}{N_{c}} & \frac{T-U}{N_{c}} & 0 \\
0 & U & 0 & 0 & 0 & 0 & 0 & \frac{U-T}{N_{c}} & -\frac{T}{N_{c}} \\
0 & 0 & T & 0 & 0 & 0 & -\frac{U}{N_{c}} & \frac{T-U}{N_{c}} & 0 \\
0 & 0 & 0 & (T+U) & 0 & 0 & \frac{U}{N_{c}} & 0 & \frac{T}{N_{c}} \\
0 & 0 & 0 & 0 & U & 0 & 0 & \frac{U-T}{N_{c}} & -\frac{T}{N_{c}} \\
0 & 0 & 0 & 0 & 0 & (T+U) & \frac{U}{N_{c}} & 0 & \frac{T}{N_{c}} \\
\frac{T-U}{N_{c}} & 0 & \frac{T-U}{N_{c}} & \frac{T}{N_{c}} & 0 & \frac{T}{N_{c}} & 2 T & 0 & 0 \\
-\frac{U}{N_{c}} & -\frac{T}{N_{c}} & -\frac{U}{N_{c}} & 0 & -\frac{T}{N_{c}} & 0 & 0 & 0 & 0 \\
0 & \frac{U-T}{N_{c}} & 0 & \frac{U}{N_{c}} & \frac{U-T}{N_{c}} & \frac{U}{N_{c}} & 0 & 0 & 2 U
\end{array}\right),
$$

where $T=\ln \frac{1}{2}\left(1-\cos \theta^{*}\right), U=\ln \frac{1}{2}\left(1+\cos \theta^{*}\right)$ in terms of the c.m. scattering angle $\theta^{*}$. The same matrix appears in explicit two-loop calculations of elastic gluon-gluon 
amplitudes [11], where it controls the single poles in dimensional regularization. The all-orders nature of this relationship has been demonstrated in [31].

\section{3 $Q_{T}$ and joint resummation}

If threshold resummation strengthens our confidence in cross sections that we can calculate to fixed order, there is a class of hadronic cross sections for which resummation is absolutely necessary even to get started $[33,34]$. The most familiar of these are the hadronic electroweak annihilation cross sections at measured transverse momentum $\left(Q_{T}\right)$ for the production of an electroweak boson $B(Q)$ (virtual photon, $\mathrm{W}, \mathrm{Z}$ or Higgs, of mass $Q$ ). In the underlying processes $q \bar{q} \rightarrow B(Q)$ or $g g \rightarrow B(Q)$ (the latter through a fermion loop), the limit $Q_{T} \rightarrow 0$ is singular in much the same way as $1-z \rightarrow 0$ at partonic threshold, Eq. (7), with plus distributions in $Q_{T}^{2}$ up to $\left(1 / Q_{T}^{2}\right) \ln ^{2 k-1}\left(Q_{T}^{2} / Q^{2}\right)$ at order $\alpha_{s}{ }^{k}$. Indeed, these two limits are closely connected and involve the same underlying factorization, and the resummation necessary for logarithms in transverse momentum is compatible with threshold resummation [28, 35]. Again, the result for the factorized/resummed cross section exponentiates in transform space, in this case a combination of Mellin $(N)$ and Fourier ( $b$, 'impact parameter') transforms. The 'jointly resummed' physical cross section is then approximated by a double inverse transform,

$$
\begin{aligned}
\frac{d \sigma^{\mathrm{res}}}{d Q^{2} d Q_{T}^{2}} \sim \sum_{a} & \sigma_{0}^{a \bar{a} \rightarrow B(Q)} \int_{C} \frac{d N}{2 \pi i} \tau^{-N} \int_{C_{b}} \frac{d^{2} b}{(2 \pi)^{2}} \mathrm{e}^{i \vec{Q}_{T} \cdot \vec{b}} \\
& \times \mathcal{C}_{a}(Q, b, N) \mathrm{e}^{E_{a \bar{a}}(N, b, Q)} \mathcal{C}_{\bar{a}}(Q, b, N)
\end{aligned}
$$

where the PDFs are included in the factors

$$
C_{a}(Q, b, N, \mu)=\sum_{j} C_{a / j}\left(N, \alpha_{s}(\mu)\right) f_{j}(N, Q /(N+b Q)) .
$$

The leading logarithmic corrections are of much the same form as in threshold resummation, Eq. (9),

$$
E_{a \bar{a}}(N, b, Q) \sim 2 \int_{[Q /(N+b Q)]^{2}}^{Q^{2}} \frac{d m^{2}}{m^{2}} A_{a}\left(\alpha_{s}(m)\right) \ln \left(\frac{m}{Q}\right),
$$

in terms of the same anomalous dimensions $A_{a}\left(\alpha_{s}\right)$. A resummed cross section calculated from (12) is compared to Tevatron data for $\mathrm{Z}$ production in Fig. 5. Similar good fits are found on the basis of $Q_{T}$-resummation alone $[33,34]$. We note in the figure a general resemblance to the heavy jet mass distribution of Fig. 2, where now the exclusive limit is at $Q_{T}=0$, which has the kinematics of quark-antiquark annihilation without gluon radiation. There is a need for modest [34, 36] but nonzero 'power' corrections, as shown in the figure. 


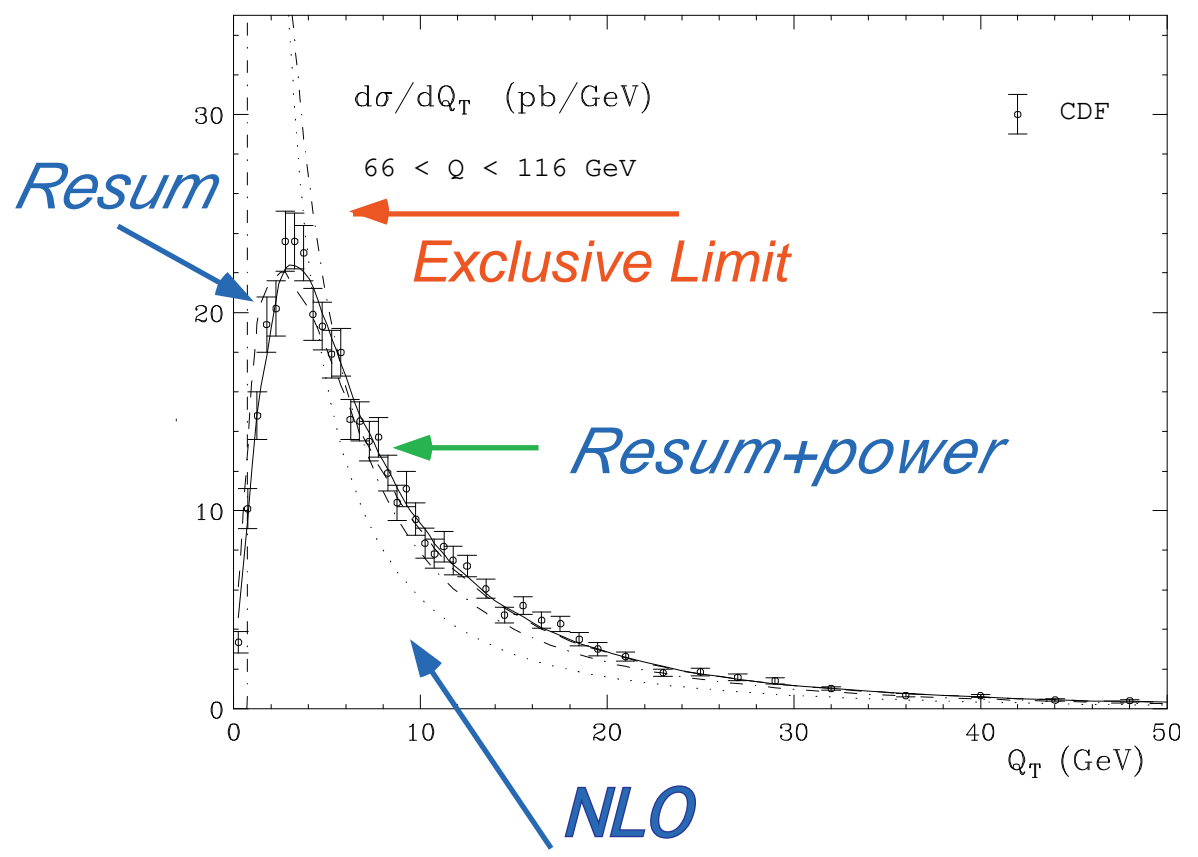

Figure 5: Jointly-resummed cross section $d \sigma / d Q_{T}$ for $\mathrm{Z}$ production at the Tevatron, from Ref. [36].

\section{Power Corrections and Event Shape Functions}

Examining Figs. 2 and 5 we see that for differential distributions, power corrections are necessary to bring even resummed cross sections into agreement with data in the exclusive limit. In the range where the kinematics are far from elastic, fixed-order perturbation theory is enough, but as we climb the curves of these figures toward the elastic limit, the cross section rises and we move into a region where first resummation, and then nonperturbative corrections dominate. The theory itself knows no strict boundary between perturbative and nonperturbative regimes. It is therefore natural to analyze perturbation theory to see if it gives signals of its own incompleteness, and to seek hints on how to supplement perturbative predictions with nonperturbative information. The ultimate goal is to construct a single theory that bridges the gap between short- and long-distance dynamics.

\subsection{The operator product expansion}

The approach that I will describe below begins with the simplest one-scale problem, the total cross section in $\mathrm{e}^{+} \mathrm{e}^{-}$annihilation [37]. The total cross section is related by the optical theorem to the imaginary part of the vacuum polarization for electroweak 
currents,

$$
\pi\left(Q^{2}\right)=-\frac{i}{3 Q^{2}} \int d^{4} x \mathrm{e}^{-i q \cdot x}\left\langle 0\left|T j^{\mu}(0) j_{\mu}(x)\right| 0\right\rangle,
$$

whose behavior at large $Q^{2} \equiv q^{2}$ is determined by an operator product expansion:

$$
\begin{aligned}
\left\langle 0\left|j^{\mu}(0) j_{\mu}(x)\right| 0\right\rangle & =\frac{1}{x^{6}} \tilde{C}_{0}\left(x^{2} \mu^{2}, \alpha_{s}(\mu)\right) \\
& +\frac{1}{x^{2}} \tilde{C}_{F^{2}}\left(x^{2} \mu^{2}, \alpha_{s}(\mu)\right)\left\langle 0\left|F_{\mu \nu} F^{\mu \nu}(0)\right| 0\right\rangle+\ldots
\end{aligned}
$$

Here, I have shown only the contributions of the leading (identity) operator and of the gluon condensate $F^{2}$. Neglecting masses, perturbation theory alone would give only the leading term, even though the full theory demands both (and more). The question we ask is whether we could have discovered the need for the nonleading operators from perturbative QCD alone.

The answer is yes, and the reasoning is illustrated in Fig. 6, which shows graphical configurations where loop momenta $k$ are vanishingly small for the vacuum polarization and for the gluon condensate treated as a local operator. Diagram-by-diagram, each such configuration is a tiny 'corner' of loop momentum space, where one or more loops has low momentum, giving a finite contribution to the infrared safe integrals. For example, if the loop momentum is required to have $k^{2}<\kappa^{2}$, for some cutoff $\kappa$, this contribution is suppressed by $\left(\kappa^{2} / Q^{2}\right) \alpha_{s}(\mu)$ compared to the full diagram. It is thus indeed power suppressed. Using gauge invariance and the renormalization group, however, we can show that summing over the self-energies indicated in the figure has the effect of making the coupling run and of giving an even greater suppression. For the vacuum polarization, we find momentum-space integrals of the general form

$$
\begin{aligned}
& C_{0}^{\mathrm{IR}}(Q, \kappa)=\frac{1}{Q^{4}} H(Q) \int_{0}^{\kappa^{2}} d k^{2} k^{2} \alpha_{s}\left(k^{2}\right) \\
& =\frac{1}{Q^{4}} H(Q) \int_{0}^{\kappa^{2}} d k^{2} k^{2} \frac{\alpha_{s}\left(Q^{2}\right)}{1+\left(\frac{\alpha_{s}\left(Q^{2}\right)}{4 \pi}\right) \beta_{0} \ln \left(k^{2} / Q^{2}\right)},
\end{aligned}
$$

with $H(Q)$ an infrared safe function free of all contributions where a single loop momentum vanishes. $H(Q)$ represents the lower, contracted part of the diagram on the left of Fig. 6. Up to this factor, the expression for the right-hand figure, corrections to the condensate, is identical. A generalization of Eq. (17) is valid to all orders in perturbation theory, and controls all logarithms in the loop momentum $k$, up to the next power, $Q^{-6}$ [37]. We will call an expression at this level of accuracy, all logs at a given nonleading power, an 'internal resummation' at that power.

From (17) we learn first, that the internally-resummed contributions are singular for very small $k^{2}$, from high order diagrams. Correspondingly, it is easy to verify that 
the $n$th order of the reexpansion of Eq. (17) in $\alpha_{s}(Q)$ is proportional to $n$ !. We also learn that these infrared-sensitive contributions in the vacuum polarization generate exactly the perturbative expansion of the gluon condensate. We conclude that the perturbative vacuum polarization requires the presence of a nonperturbative gluon condensate, which should replace its misleading high-order infrared behavior.
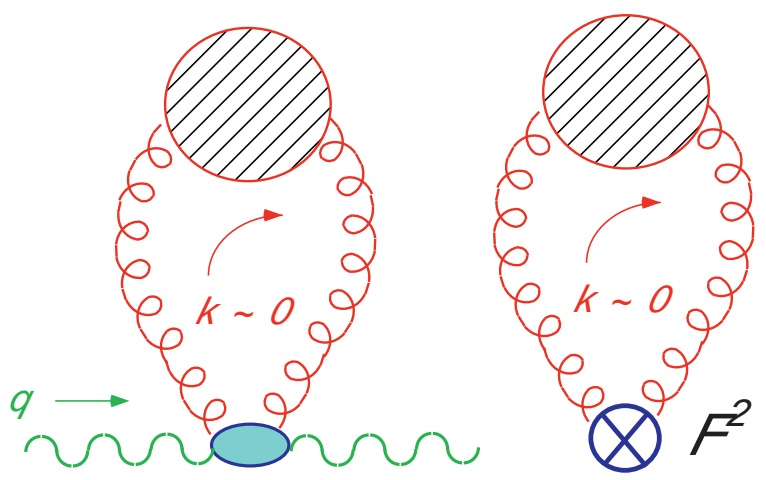

Figure 6: Sources of infrared sensitivity in perturbation theory for $C_{0}$ (left) and $\left\langle F^{2}\right\rangle$ (right).

The foregoing steps may be summarized as an 'axiom of substitution', in which the infrared-sensitive, nonconvergent portion of an internally-resummed perturbative quantity is replaced by a matrix element with the same perturbative infrared expansion:

$$
\begin{aligned}
C_{\mathrm{PT}} \rightarrow C_{\mathrm{PT}}^{\mathrm{reg}} & \left(Q^{2} / \mu^{2}, \kappa / Q, \alpha_{s}(\mu)\right) \\
& +\frac{1}{Q^{4}} C_{F^{2}}(Q, \kappa) \alpha_{s}\left\langle 0\left|F^{2}(0)\right| 0\right\rangle(\kappa) .
\end{aligned}
$$

Here, $C_{\mathrm{PT}}^{\mathrm{reg}}$ is perturbation theory subtracted in its infrared-sensitive limit. As anticipated, the nonconvergence of perturbation theory implies the need for a new, infrared regularization. For the vacuum polarization the cost is a new nonperturbative parameter $\left(\left\langle F^{2}\right\rangle\right)$, necessary to define the theory at the level of $1 / Q^{4}$ relative to the leading behavior. But, because it is implicit in perturbation theory, this new parameter is at the same time a reward of the analysis.

\subsection{Power corrections in semi-inclusive cross sections}

The viewpoint described above for the operator product expansion has been implemented, in various ways, to a variety of infrared safe cross sections and hard-scattering functions [38]. Of particular interest are resummed hard scattering functions like Eqs. (9) and (14), which exhibit the same integral over the scale of the coupling as for the vacuum polarization (17). The application of these methods to event shape cross sections [39] sheds light on the transition from short- to long-distance dynamics in the 
formation of final states in QCD. This section will sketch the ideas behind these applications, and also touch on internal resummation in this context. Such questions are most easily addressed for event shapes of two-jet cross sections in $\mathrm{e}^{+} \mathrm{e}^{-}$annihilation, although their application is much wider [40].

We consider inclusive cross sections defined by event shapes, $S\left(\left\{p_{i}\right\}\right)$,

$$
\frac{d \sigma}{d \mathcal{S}}=\frac{1}{2 Q^{2}} \sum_{n} \int_{P S(n)}\left|M_{n}\left(\left\{p_{i}\right\}\right)\right|^{2} \delta\left(S\left(\left\{p_{i}\right\}\right)-\mathcal{S}\right)
$$

at c.m. energy $Q$, computed from QCD matrix elements $M_{n}\left(\left\{p_{i}\right\}\right)$ integrated over $n$ particle phase space, $P S(n)$. This event shape cross section is infrared safe if $S\left(\left\{p_{i}\right\}\right)$ is a smooth function of final state momenta, $p_{i}$, that is unchanged by soft parton emission and collinear rearrangements, as illustrated in Fig. 7:

$$
S\left(\ldots p_{i} \ldots p_{j-1}, \alpha p_{i}, p_{j+1} \ldots\right)=S\left(\ldots(1+\alpha) p_{i} \ldots p_{j-1}, p_{j+1} \ldots\right) \text {. }
$$

This condition makes it possible to compute the cross section perturbatively, because it deemphasizes the long-time evolution of the system, over which the nearly degenerate states of Fig. 7 mix.

The study of event shapes in QCD, and more generally energy flow [41], continues a long tradition in field theory. We may draw a not too far-fetched analogy between how, late in the nineteenth century Poynting discovered the rules for energy and momentum flow in classical electrodynamics by considering the slow discharge of a condenser [42], and how, late in the twentieth century, the short-distance structure of QCD was revealed in the rapid neutralization of a quark-antiquark color dipole produced in $\mathrm{e}^{+} \mathrm{e}^{-}$ annihilation [43].

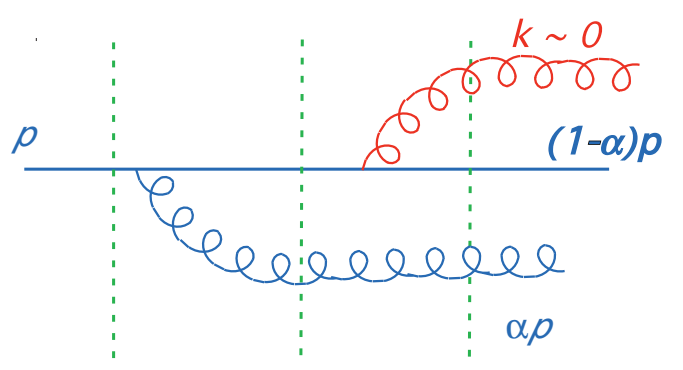

Figure 7: Soft emission and collinear rearrangements.

We restrict ourselves here to a specific set of weights, appropriate to dijet events in annihilation cross sections [44],

$$
\mathcal{S}_{a}(n)=\frac{1}{Q} \sum_{\text {all } i \in n} k_{i T} \mathrm{e}^{-\left|\eta_{i}\right|(1-a)}
$$


where the transverse momentum $k_{i T}$ and pseudorapidity $\eta_{i}$ of each particle momentum $k_{i}$ is defined relative to the axis that minimizes the specific function $\mathcal{S}_{0}(n)$ for state $n$. The variable $a$ takes on any value less than two. The particular choice $a=0$ is $\mathcal{S}_{0}=1-T$ with $T$ the thrust, while $a=1$ is known as the jet broadening [45].

For these dijet event shapes, sensitivity to long times can be adjusted by studying the Laplace transform [39, 45],

$$
\tilde{\sigma}_{a}(N)=\frac{1}{\sigma_{\text {tot }}} \int_{0}^{1} d \mathcal{S}_{a} \mathrm{e}^{-N \mathcal{S}_{a}} \frac{d \sigma}{d \mathcal{S}_{a}} .
$$

Choosing $N$ large, we select states with small $\mathcal{S}_{a}$, which are sensitive to long-time behavior (roughly, times of order $1 / S_{a} Q$ ), corresponding to low jet masses in dijet events. As illustrated by Fig. 2, however, power corrections are important at low jet masses. We thus ask what the reasoning described above for the operator product expansion can tell us about the transformed cross sections, especially at large $N$ in Eq. (22).

At low values of $\mathcal{S}_{a}$, these differential event shape cross sections show the same double logarithmic behavior discussed above for threshold resummation, and obey a similar resummation in Laplace transform space [44, 45], which is given to leading logarithm, accompanied by effects of the running coupling, by

$$
\begin{aligned}
\tilde{\sigma}_{a}(N, Q) \sim \exp \left\{2 \int_{0}^{Q^{2}} \frac{d p_{T}^{2}}{p_{T}^{2}}\right. & A_{q}\left(\alpha_{s}\left(p_{T}\right)\right) \int_{p_{T}^{2} / Q^{2}}^{p_{T} / Q} \frac{d y}{y} \\
\times & {\left.\left[\mathrm{e}^{-N\left(p_{T} / Q\right)^{a} y^{1-a}}-1\right]\right\}, }
\end{aligned}
$$

in terms of the same Sudakov anomalous dimension as above, $A_{q}\left(\alpha_{s}\right)=C_{F}\left(\alpha_{s} / \pi\right)+\ldots$.

We can use Eq. (23) to suggest the structure of power corrections in differential cross sections, as described above for the total annihilation cross section. To do so, we split up the $p_{T}$ integral into 'perturbative' and 'soft' ranges, separated at a factorization scale $\kappa$ [46]. If we expand the exponent of (23) in the soft range, we find

$$
\begin{gathered}
\ln \tilde{\sigma}_{a}(N, Q)=\ln \tilde{\sigma}_{a, \mathrm{PT}}(N, Q, \kappa) \\
+\frac{2}{1-a} \sum_{n=1}^{\infty} \frac{1}{n n !}\left(-\frac{N}{Q}\right)^{n} \int_{0}^{\kappa^{2}} \frac{d p_{T}^{2}}{p_{T}^{2}} p_{T}^{n} A\left(\alpha_{s}\left(p_{T}\right)\right)\left[1-\left(\frac{p_{T}}{Q}\right)^{n(1-a)}\right] \\
\equiv \ln \tilde{\sigma}_{a, \mathrm{PT}}(N, Q, \kappa)+\ln \tilde{f}_{\mathcal{S}_{a}}(N / Q)+\mathcal{O}\left(\frac{N}{Q^{2-a}}\right) .
\end{gathered}
$$

In the second line we organize the entire contribution from the soft region into a single function $\tilde{f}_{\mathcal{S}_{a}}(N / Q)$, with corrections relatively suppressed by powers in $1 / Q^{1-a}$ (assuming for now that $a<1)$. Taking the inverse transform of $(24)$ we arrive at an expression for the physical cross section as a convolution of the perturbative cross section with 
what is known as an event shape function [46],

$$
\frac{d \sigma}{d \mathcal{S}_{a}}=\int_{0}^{\mathcal{S}_{a} Q} d \epsilon f_{\mathcal{S}_{a}}(\epsilon) \frac{d \sigma_{a, \mathrm{PT}}\left(\mathcal{S}_{a}-\epsilon / Q\right)}{d \mathcal{S}_{a}}+\mathcal{O}\left(\frac{1}{\mathcal{S}_{a} Q^{2-a}}\right)
$$

The event shape function $f_{\mathcal{S}_{a}}$ is independent of $Q$, so that a fit to data at $Q=m_{Z}$ is sufficient to predict the differential cross section at any $Q$, including all integer powers of $N / Q$ in moment space [10], which translates to all integer powers of $1 / \mathcal{S}_{a} Q$ at fixed $Q$. This is the kind of formalism necessary to confront data like those in Fig. 2 above. Analogous functions find important applications in the physics of b quarks [47]. A recent example appled to the heavy jet mass, closely related to $a=0$, is shown in Fig. 8 from Ref. [48]. Beyond the $a=0$, the shape functions derived in this fashion for choices of $a, b<1$ possess a surprisingly simple relation,

$$
\ln \tilde{f}_{\mathcal{S}_{a}}(N / Q)=\frac{1-b}{1-a} \ln \tilde{f}_{\mathcal{S}_{b}}(N / Q),
$$

with corrections as in (25). For $2>a>1$, on the other hand, we encounter the interesting situation noted first in Ref. [49], where the leading power correction becomes fractional, and larger than $1 / Q$.

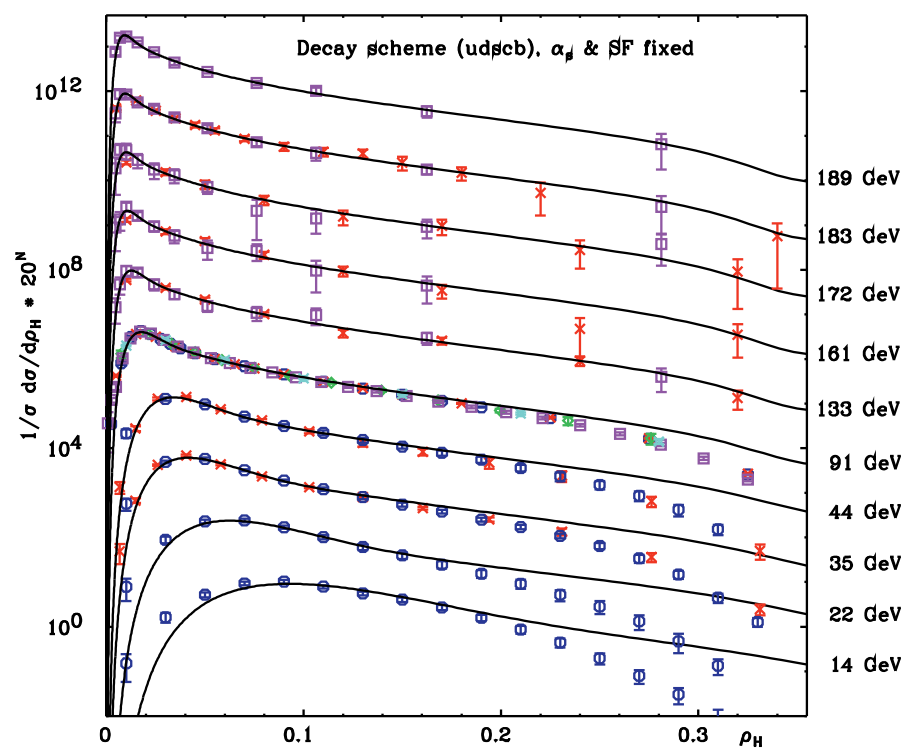

Figure 8: Event shape-based fit to the heavy jet mass at various energies, from Ref. [48].

The next question we should ask, however, is whether Eqs. (23) and (26) hold beyond leading and (suitably generalized [45]) next-to-leading logarithmic resummation, and in particular whether these event shapes can be internally resummed to all 
orders in $1 / \mathcal{S}_{a} Q$. Rather than try to answer this question for the full theory, we will follow Ref. [46], and consider the 'eikonal' cross section, in which the primary quarkantiquark pair and the partons collinear to them are all replaced by nonabelian phase operators. These are Wilson lines, represented by products of exponentials of the gauge field ordered along lightlike paths,

$$
W=T\left(\Phi_{\beta_{1}}^{(q)}(\infty, 0) \Phi_{\beta_{2}}^{(\bar{q})}(\infty, 0)\right)
$$

with

$$
\Phi_{\beta_{i}}^{(i)}(\lambda, x)=\mathcal{P} \exp \left(-i g \int_{0}^{\lambda} d \lambda^{\prime} \beta_{i} \cdot A^{(i)}\left(\lambda^{\prime} \beta_{i}+x\right)\right),
$$

where $A^{(i)}$ is the gluon field in representation $i$. In these terms, the eikonal cross sections at fixed values of event shapes $\mathcal{S}_{a}$ may be written as a sum over states, $n$ :

$$
\frac{1}{\sigma_{\text {tot }}} \frac{d \sigma^{\text {eikonal }}}{d \mathcal{S}_{a}}=\sum_{n} \delta\left(\mathcal{S}_{a}-\mathcal{S}_{a}(n)\right)\left\langle 0\left|W^{\dagger}\right| n\right\rangle\langle n|W| 0\rangle .
$$

These cross sections have the advantage that many of their properties may be analyzed to all orders in perturbation theory using combinatoric methods [50]. Here I will simply quote the result of such an analysis.

As in Eq. (23), the all-orders eikonal approximation to the cross section can be written in transform space in terms of a function in which $\alpha_{s}$ runs with an integration variable, which we may think of as a transverse momentum,

$$
\ln \tilde{\sigma}_{a}(N, Q)=\int_{0}^{Q^{2}} \frac{d^{2} p_{T}}{p_{T}^{2}} \mathcal{A}_{\mathcal{S}_{a}}\left(p_{T} / \mu, Q / \mu, \alpha_{s}(\mu), N\right),
$$

where the function $\mathcal{A}_{\mathcal{S}_{a}}$ is infrared safe and renormalization group invariant $\left(d \mathcal{A}_{\mathcal{S}_{a}} / d \mu=\right.$ $0)$. As an example, consider the case $a=1$, jet broadening, for which we can write

$$
\begin{aligned}
& \mathcal{A}_{\mathcal{S}_{1}}\left(p_{T} / \mu, Q / \mu, \alpha_{s}(\mu), N\right) \\
& =\sum_{n} \int_{P S(n)} \mathcal{W}_{n}\left(\frac{k_{i} \cdot k_{j}}{\mu^{2}}, \frac{k_{i} \cdot \beta_{1} k_{j} \cdot \beta_{2}}{\mu^{2} \beta_{1} \cdot \beta_{2}}, \alpha_{s}(\mu)\right) \\
& \quad \times \delta^{2}\left(p_{T}-\sum_{i \in n} k_{i, T}\right) \delta\left(\eta_{n}\right) \ln \left(Q / p_{T}\right)\left[\exp \left\{-\frac{N}{Q} p_{T}\right\}-1\right],
\end{aligned}
$$

where the $k_{i}$ denote particle momenta in final state $n$, and where $\eta_{n}$ is the rapidity of the total momentum of state $n$. Boost invariance requires that the functions $\mathcal{W}_{n}$ [50] depend only on the arguments shown. For other event shapes, such as the thrust, slightly more 
elaborate expressions are necessary. In each case, however, a meaningful factorization of the infrared, strong-coupling dynamics from the perturbative region is possible. Eq. (31), although valid for all orders and logarithms, applies only to the eikonal cross section. Nevertheless, it appears that the way is open for the all-orders analysis of the relation between perturbation theory and power corrections in event shape functions and related parameterizations, beyond the limitations of next-to-leading logarithm.

\section{Summary}

Short-distance, single-scale observables in QCD are reasonably well understood, which is one reason why we have such confidence that QCD is a true theory of the strong interactions. The developing technology of next-to-next-to-leading order, coupled with a sophisticated approach to parton distribution uncertainties, should make possible precision for selected observables at the level of one percent.

At the same time, we are far from a full understanding of multi-scale observables, which lead us from perturbative to nonperturbative dynamics. Power corrections and event shape functions for infrared safe differential distributions may be identified by an extension of the reasoning that leads to the operator product expansion for the total $\mathrm{e}^{+} \mathrm{e}^{-}$annihilation cross section. By exploring concepts like internal resummation, we may find a perturbative window to the formation of final states, and gain insight into the transition between short- and long-distance degrees of freedom in quantum chromodynamics.

\section{Acknowledgements}

I thank the organizers of TH2002 and of the 26th Johns Hopkins Workshop for their invitations, and for making possible my participation. This work was supported in part by the National Science Foundation grant PHY0098527.

\section{References}

[1] E.L. Berger et al., Summary, Working Group on QCD and Strong Interactions, APS/DPF/DPB Summer Study on the Future of Particle Physics (Snowmass 2001), [hep-ph/0201146].

[2] G. Sterman and S. Weinberg, Phys. Rev. Lett. 39 (1977) 1436; A. de Rújula, J. Ellis, E.G. Floratos and M.K. Gaillard, Nucl. Phys. B 138 (1978) 387.

[3] J.C. Collins and D.E. Soper, Ann. Rev. Nucl. Part. Sci. 37 (1987) 383; J.C. Collins, D.E. Soper and G. Sterman, in Perturbative Quantum Chromodynamics, ed. A.H. Mueller (World Scientific, Singapore, 1989), p. 1. 
[4] C.-H.V. Chang and H.-n. Li, Phys. Rev. D 55 (1977) 5577, [hep-ph/9607214]; M. Beneke, G. Buchalla, M. Neubert and C.T. Sachrajda, Phys. Rev. Lett. 83 (1999) 1914, [hep-ph/9905312]; C.W. Bauer, D. Pirjol and I.W. Stewart, Phys. Rev. Lett 87 (2001) 201806, [hep-ph/0107002].

[5] A.H. Mueller, Phys. Rep. 73 (1981) 237.

[6] J.C. Collins, D.E. Soper and G. Sterman, Nucl. Phys. B 261 (1985) 104; ibid., B 308, (1988) 833; G.T. Bodwin, Phys. Rev. D 31 (1985) 2616, E. ibid. D34 (1986) 3932 .

[7] C.W. Bauer, D. Pirjol and I.W. Stewart,, Phys. Rev. D 65 (2002) 054022, [hepph/0109045]; C.W. Bauer et al., Phys. Rev. D66 (2002) 014017, [hep-ph/0202088].

[8] G. Altarelli and G. Parisi, Nucl. Phys. B 126 (1977) 298; V.N. Gribov and L.N. Lipatov, Sov. J. Nucl. Phys. 15 (1972) 438, 675; Y.L. Dokshitzer, Sov. Phys. JETP 46 (1977) 641.

[9] ZEUS Collaboration (S. Chekanov et al.), Eur. Phys. J. C 21 (2001) 443, [hepex/0105090].

[10] G.P. Korchemsky and S. Tafat, JHEP 0010 (2000) 010, [hep-ph/0007005].

[11] V.A. Smirnov, Phys. Lett. B 460 (1999) 367, [hep-ph/9905323]; V.A. Smirnov and O.L. Veretin, Nuc. Phys. B566 (2000) 469, [hep-ph/9907385]; J.B. Tausk, Phys. Lett. B 469 (1999) 225, [hep-ph/9909506]; Z. Bern, L.J. Dixon and D.A. Kosower, JHEP 0001 (2000) 027, [hep-ph/0001001]; C. Anastasiou et al., Nucl. Phys. B 580 (2000) 577, [hep-ph/0003261]; Z. Bern, L.J. Dixon and A. Ghinculov, Phys. Rev. D 63 (2001) 053007, [hep-ph/0010075] C. Anastasiou, E.W.N. Glover, C. Oleari and M.E. Tejeda-Yeomans, Nucl. Phys. B 605 (2001) 486, [hep-ph/0101304].

[12] C. Oleari, 37th Rencontres de Moriond on the Cosmological Model, Les Arcs, France, March 2002, [hep-ph/0205184]; T. Gehrmann, 6th International Symposium on Radiative Corrections and 6th Zeuthen Workshop on Elementary Particle Theory, Kloster Banz, Germany, Sept. 2002, [hep-ph/0210157]; A.I. Davydychev and V.A. Smirnov, 8th International Workshop on Advanced Computing and Analysis Techniques in Physics Research (ACAT 2002), Moscow, Russia, June 2002, [hep-ph/0210171].

[13] P.N. Burrows, APS/DPF/DPB Summer Study on the Future of Particle Physics (Snowmass 2001), [hep-ex/0112036].

[14] L.W. Garland et al., Nucl. Phys. B 642 (2002) 227, [hep-ph/0206067]; S. Moch, P. Uwer and S. Weinzierl, 6th International Symposium on Radiative Corrections and 6th Zeuthen Workshop on Elementary Particle Theory, Kloster Banz, Germany, Sept. 2002, [hep-ph/0211156]. 
[15] S.A. Larin, P. Nogueira, T. van Ritbergen and J.A.M. Vermaseren, Nucl. Phys. 492 (1997) 338, [hep-ph/9605317].

[16] A.L. Kataev, A.V. Kotikov, G. Parente and A.V. Sidorov, Phys. Lett. B417 (1998) 374, [hep-ph/9706534]; J. Santiago and F.J. Yndurain, Nucl. Phys. B 563 (1999) 45, [hep-ph/9904344]; W.L. van Neerven and A. Vogt, Nucl. Phys. B 603 (2001) 42, [hep-ph/0103123]; A.D. Martin, R.G. Roberts, W.J. Stirling and R.S. Thorne, Phys. Lett. B 531 (2002) 216, [hep-ph/0201127].

[17] S. Moch, J.A.M. Vermaseren and A. Vogt, Nucl. Phys. B 646 (2002) 181, [hepph/0209100]; C.F. Berger, Phys. Rev. D 66 (2002) 116002, [hep-ph/0209107].

[18] FNAL E866/NuSea Collaboration (R.S. Towell et al.), Phys. Rev. D 64 (2001) 052002, [hep-ex/0103030].

[19] J. Pumplin et al. JHEP 0207 (2002) 012, [hep-ph/0201195].

[20] A.D. Martin, R.G. Roberts, W.J. Stirling and R.S. Thorne, Eur. Phys. J. C 23 (2002) 73, [hep-ph/0110215].

[21] S. Alekhin, Eur. Phys. J. C 10 (1999) 395, [hep-ph/9611213]; W.T. Giele and S. Keller, Phys. Rev. D 58 (1998) 094023, [hep-ph/9803393]; M. Botje, Eur. Phys. J. C 14 (2000) 285, [hep-ph/9912439]; V. Periwal, Phys. Rev. D 59 (1999) 094006, [hep-ph/9808474]; S. Kuhlmann et al., Phys. Lett. B 476 (2000) 291, [hepph/9912283]; J. Pumplin et al., Phys. Rev. D 65 (2002) 014013, [hep-ph/0101032]; ibid. 014012, [hep-ph/0101051]; W.T. Giele, S.A. Keller and D.A. Kosower, [hepph/0104052]; W. Giele et al, The QCD/SM Working Group Report, Workshop on Physics at TeV Colliders, Les Houches, 2001, [hep-ph/0204316].

[22] CDF Collaboration and D0 Collaboration (Sally Seidel for the collaborations), 37th Rencontres de Moriond on QCD and Hadronic Interactions, Les Arcs, France, 2002, [hep-ex/0205013].

[23] M. Cacciari and P. Nason, Phys. Rev. Lett. 89 (2002) 122003, [hep-ph/0204025]; B.A. Kniehl, [hep-ph/0211008].

[24] R.V. Harlander and W.B. Kilgore, Phys. Rev. Lett. 88 (2002) 201801, [hepph/0201206]; JHEP 0210 (2002) 017, [hep-ph/0208096]; C. Anastasiou and K. Melnikov, Nucl. Phys. B 646, (2002) 220, [hep-ph/0207004]; [hep-ph/0208115].

[25] G. Sterman, Nucl. Phys. B281 (1987) 310; S. Catani and L. Trentadue, Nucl. Phys. B327 (1989) 323; ibid., B353 (1991) 183; E.L. Berger and H. Contopanagos, Phys. Rev. D 57 (1998) 253, [hep-ph/9706206]; R. Bonciani, S. Catani, M.L. Mangano and P. Nason Nucl. Phys. B 529 (1989) 424, [hep-ph/9801375]; N. Kidonakis and R. Vogt Phys. Rev. D 59 (1999) 074014, [hep-ph/9806526]; S. Catani et al., JHEP 
9903 (1999) 025, [hep-ph/9903436]; N. Kidonakis and J.F Owens, Phys. Rev. D 61 (2000) 094004, [hep-ph/9912388]; ibid., D63, 054019 (2001), [hep-ph/0007268] G. Sterman and W. Vogelsang, JHEP 0102 (2001) 016, [hep-ph/0011289].

[26] H. Contopanagos and G. Sterman, Nucl. Phys. B 419 (1994) 77, [hep-ph/9310313]; E.L. Berger and H. Contopanagos, Ref. [25].

[27] S. Catani, M.L. Mangano, P. Nason and L. Trentadue, Nucl. Phys. B478 (1996) 273, [hep-ph/9604351].

[28] E. Laenen, G. Sterman, and W. Vogelsang, Phys. Rev. Lett. 84 (2000) 4296, [hep$\mathrm{ph} / 0002078]$.

[29] N. Kidonakis and J.F. Owens, Ref. [25]; N. Kidonakis, E. Laenen, S. Moch and R. Vogt, Phys. Rev. D 64 (2001) 114001, [hep-ph/0105041].

[30] S. Catani, Phys. Lett. B 427 (1998) 161, [hep-ph/9802439].

[31] G. Sterman and M.E. Tejeda-Yeomans, [hep-ph/0210130].

[32] N. Kidonakis, G. Oderda and G. Sterman, Nucl. Phys. B 531 (1998) 365, [hep$\mathrm{ph} / 9803241]$.

[33] Y.L. Dokshitzer, D.I. D'Yakonov and S.I. Troyan, Phys. Lett. 79B (1978) 269; G. Parisi and R. Petronzio, Nucl. Phys. B 154 (1979) 427; G. Altarelli, R.K. Ellis, M. Greco and G. Martinelli, Nucl. Phys. B 246 (1984) 12; J.C. Collins and D.E. Soper, Nucl. Phys. B 193 (1981) 381; E: ibid B 213 (1983) 545; Nucl. Phys. B 197 (1982) 446; J.C. Collins, D.E. Soper and G. Sterman, Nucl. Phys. B 250 (1985) 199; C.T.H. Davies and W.J. Stirling, Nucl. Phys. B 244 (1984) 337; C.T.H. Davies, W.J. Stirling and B.R. Webber, Nucl. Phys. B 256 (1985) 413; P.B. Arnold and R.P. Kauffman, Nucl. Phys. B 349 (1991) 381; G.A. Ladinsky and C.-P. Yuan, Phys. Rev. D 50 (1994) 4239, [hep-ph/9311341]; F. Landry, R. Brock, G. Ladinsky and C.-P. Yuan, Phys. Rev. D 63 (2001) 013004, [hep-ph/9905391]; C. Balazs and C.P. Yuan, Phys. Rev. D 56 (1997) 5558, [hep-ph/9704258].

[34] J.-w. Qiu and X.-f. Zhang, Phys. Rev. Lett. 86 (2001) 2724, [hep-ph/0012058]; Phys. Rev. D 63 (2001) 114011, [hep-ph/0012348].

[35] H.-N. Li, Phys. Lett. B 454 (1999) 328, [hep-ph/9812363].

[36] A. Kulesza, G. Sterman and W. Vogelsang, Phys. Rev. D 66 (2002) 014011, [hep$\mathrm{ph} / 0202251]$.

[37] A.H. Mueller, Nucl. Phys. B 250 (1985) 327; in QCD: 20 Years Later, proceedings of Workshop on QCD, Aachen, Germany, June 1992, eds. P.M. Zerwas and H.A. Kastrup (World Scientific, Singapore, 1993), p. 162; Phys. Lett. B 308 (1993) 355. 
[38] H. Contopanagos and G. Sterman, Ref. [26]; A.V. Manohar and M.B. Wise, Phys. Lett. B 344 (1995) 407, [hep-ph/9406392]; B.R. Webber, Phys. Lett. B 339 (1994) 148, [hep-ph/9408222]; G.P. Korchemsky and G. Sterman, Nucl. Phys. B 437 (1995) 415, [hep-ph/9411211]; and 30th Renconters de Moriond: QCD and High Energy Hadronic Interactions, ed. J. Trân Thanh Vân, Editions Frontiers, (Paris, 1995), p. 383, [hep-ph/9505391]; Y.L. Dokshitzer and B.R. Webber, Phys. Lett. B 352 (1995) 451, [hep-ph/9504219]; R. Akhoury and V.I. Zakharov, Nucl. Phys. B 465 (1996) 295, [hep-ph/9507253]; M. Beneke, V.M. Braun and L. Magnea, Nucl. Phys. B497 (1997) 297, [hep-ph/9701309]; M. Beneke and V.M. Braun, in the Boris Ioffe Festschrift, At the Frontier of Particle Physics, Handbook of QCD, ed. M. Shifman (World Scientific, Singapore, 2001), vol. 3, p. 1719, [hep-ph/0010208].

[39] G.P. Korchemsky and G. Sterman, in proceedings of 30th Rencontres de Moriond: QCD and High Energy Hadronic Interactions, 1995, [hep-ph/9505391]; Y.L. Dokshitzer and B.R. Webber, Phys. Lett. B404 (1997) 321, [hep-ph/9704298].

[40] A. Banfi, Y.L. Dokshitzer, G. Marchesini and G. Zanderighi, JHEP 0103 (2001) 007, [hep-ph/0101205].

[41] N.A. Sveshnikov and F.V. Tkachov, Phys. Lett. B 382 (1996) 403, [hepph/9512370]; F.V. Tkachov, Int. J. Mod. Phys. A 12 (1997) 5411, [hepph/9601308]; C.F. Berger et al., in proceedings of the APS/DPF/DPB Summer Study on the Future of Particle Physics (Snowmass 2001), [hep-ph/0202207].

[42] J.H. Poynting, Philosophical Transactions of the Royal Society of London. For the year MDCCCLXXXIV, Vol. 175, Part II, p. 343.

[43] G. Hanson et al., Phys. Rev. Lett. 35 (1975) 1609.

[44] C.F. Berger, T. Kucs and G. Sterman, [hep-ph/0212343] and in preparation.

[45] S. Catani, G. Turnock, L. Trentadue, and B.R. Webber, Phys. Lett. B 263 (1991) 491; ibid. B 295 (1992) 269; Nucl. Phys. B 407 (1993) 3; Y.L. Dokshitzer, A. Lucenti, G. Marchesini and G. Salam, JHEP, 9801 (1998) 011, [hep-ph/9801324].

[46] G.P. Korchemsky and George Sterman, Nucl. Phys. B 555 (1999) 335, [hepph/9902341]; A.V. Belitsky, G.P. Korchemsky and G. Sterman, Phys. Lett. B 515 (2001) 297, [hep-ph/0106308].

[47] M. Neubert, Phys. Rev. D 49 (1994) 3392, [hep-ph/9311325]; G.P. Korchemsky and G. Sterman, Phys. Lett. B 340 (1994) 96, [hep-ph/9407344]; U. Aglietti et al, Phys. Lett. B 432 (1998) 411, [hep-ph/9804416]; T. Mannel and S. Recksiegel, Phys. Rev. D63 (2001) 094011, [hep-ph/0009268].

[48] E. Gardi and J. Rathsman, Nucl. Phys. B 638 (2002) 243, [hep-ph/0201019]. 
[49] A.V. Manohar and M.B. Wise, Ref. [38].

[50] G. Sterman in Perturbative Quantum Chromodynamics, ed. D.W. Duke and J.F. Owens (American Institute of Physics, New York, 1981), p. 22; J.G.M. Gatheral, Phys. Lett. B 133 (1983) 90; J. Frenkel and J.C. Taylor, Nucl. Phys. B 246 (1984) 231; C.F. Berger Ref. [17]. 